\title{
Integration of Compressive Sensing and Clustering in Wireless Sensor Networks using Block Tridiagonal Matrix Method
}

\author{
Venkat Rao Pasupuleti, Ch. Balaswamy
}

\begin{abstract}
The most dominant applications of wireless sensor networks (WSNs) is Environmental monitoring, it generally needs long time to operate. Although, the energy of inherent restriction has the bottle neck in scale of each WSN applications. This articler demonstrates the framework for an integration of compressive sensing and blocks tri-diagonal matrices (BDMs) for the clustering in WSNs that can be used as the matrices of measurement by the combination of data prediction that is involved with the compression and retrieval to achieve data processing precision and effectiveness in clustered WSNs simultaneously. On basis of the analysis theoretically, this can be designed for the implementation in number of algorithms. The proposed framework furnishes the real world data demonstration which can be utilized to get the simulation results for a solution of cost effective for the applications on basis of cluster in WSNs for environmental monitoring.
\end{abstract}

Index Terms: Compressive sensing, Data prediction, Environmental monitoring, Matrix based compression, Wireless sensor networks (WSNs)

\section{INTRODUCTION}

The deployment of sensor nodes repeatedly that collect data readings and transmit them to sinks or BSs through wireless communication in wireless senor networks. It is required to demonstrate the simple and data gathering of energy efficient method to minimize the consumption of energy of each sensor node and this is because of the capability for the computations that are limited and power of energy. The most essential factors of WSNs are the consumption of energy at the time of transmitting and receiving of data. For achieving the data compression, it has been proposed various methods that comprises of tradition for source coding and distributed source coding. The collection information in wireless sensors namely temperature, pressure, vibration, humidity, biomedical strength, pollutants and real-world monitoring[1]. The most essential factor that can determine the network lifetime frequently is the consumption of power consumption. The minimized energy resources that can be managed in $\mathrm{WN}$ by battery and life[2]. For the reduction of number of bits in information in WSNs, the compressive sensing(CS) offers for the new sampling method to minimize the consumption of power. The sensing theory of compression for sparsing of

Revised Manuscript Received on August 20, 2019.

Venkat Rao Pasupuleti*, Department of ECE, Lakireddy Balireddy College of Engineering, Mylavaram, India.

Ch. Balaswamy, Department of ECE, Gudlavalleru Engineering College, Gudlavalleru, India. signals for attaining the minimized sampling rate as a new idea for such signals in WSNs. The compression offers sensing where the information of sparse signal can be reconstructed from the linear information of measurements in minimized number of randomness in sensor networks.

The traditional schemes of data transmission are highly implemented by Multi-hop routing methods. The upstream nodes forward data from each sensor node to the receiver end on the routing tree along the path of hop by hop. All the sensor nodes remain closer to the receiver while the transmission mode and also requires the information of other distant nodes to transmit, which can be apart from transmitting their detection data, this leads to the higher loads of those nodes that remains closer[3]. The investigation has been carried out for many frameworks for sensing. Those methods that provide the practical method for sampling between them are Structured Random Matrix [4](SRM). This method has the increase in sparsity, decrease in complexity rapid property of computation with the comparative performance of sensing completely with random sensing matrices. The method which is used for exploitation for the recently introduced for the correlation patterns generally by combining the possibly of distinct bases of sacrificing from each dimension of the signal within the matrix of single basis using Kronecker compressed sensing (KCS)[5]. For the improvement of compression performance with the minimizing of expenditure over the energy of sensor with the featuring signals of normal data of WSN with characteristics. The analytical approaches of CS shown to be KCS with the single dimensional outperform [6]. Even though SRM cannot be applicable for WSNs and the maximizing of dimension in data of KCS model may result in recovery performance of degrading.

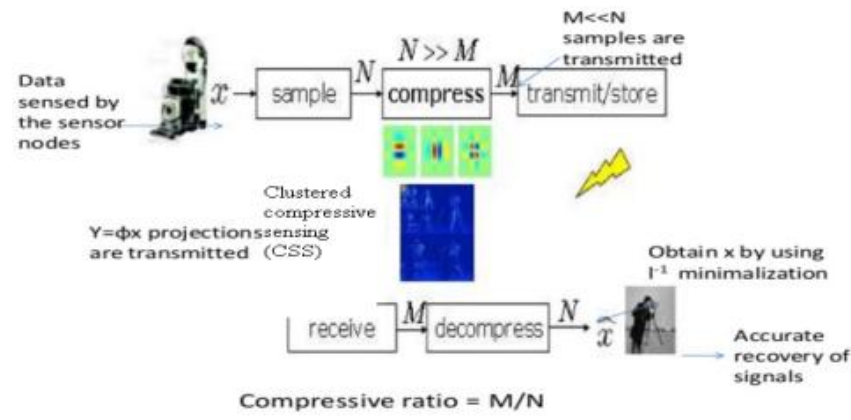

Fig. 1. Compressive sensing framework 
The consumption of Energy has been indicated mainly in following three stages: data sensing, processing of data, and data transmission. The energy consumption is usually decreased for the sensing of data and processing of data than transmission. To extend the life time of WSN's, the cost of transmission of data must be reduced. Hence by decreasing the overall consumption of energy has been essential for WSNs designing [7]. Most of the existing methods have indicated the challenge of energy efficiency in WSNs from various prospects, the conservation of energy can comprising the scheduling of sleep, control for topology, data collectors of mobile, and aggregation of data [8]. The importance on the properties of correlation for those data that are sensed in the original sensor networks, the amount of data transmitted for attaining the relative accuracy which is higher with the reduction through methods for compression at the node present in the receiving end. The proper basis DCT or wavelet domain can leads to the inherent sparsity of data for the spatial correlation [9]. It is possible to obtain the correct basis in which the sensed data from the nodes is sparse and the few data samples are not zero or equivalent.

The proposed methodology of our work based on the problems of clustering that shows the saving and energy balance regarding sensor network. This research paper shows the clustering algorithm on basis of Compressive Sensing-based clustering algorithm, a sensor network which is clustered only requires sending measurements of $\mathrm{M}$ to the base station (BS) from its clusters. The grouping of nodes forms clusters and have the hierarchy in the network which selects one cluster head $(\mathrm{CH})$ for each cluster. The information that has been sent by the nodes about the environment of corresponding $\mathrm{CH}$ and $\mathrm{CHs}$ transmits the data that are collected to the BS after data aggregation/ data fusion process. The gaining of ample interest is by Homogeneous sensor networks due to lack of increasing in the total network cost. And hence the simulation will be done by MATLAB and the results will be compared with existing HCS model.are provided.

\section{LITERATURE SURVEY}

The observation of researchers in recent years has deployed for the utilization of data aggregation methods on basis CS for enhancing the lifetime of network for the reduction of data transmission and traffic balance across the entire sensor network Feizi et al., [10] shown as the scheme of data transmission, firstly it can use sensing compression for data compression, it can be applied and simulated for the coding of network. Since these two methods are not dependent among themselves, this cannot be utilized fully for the combination of two algorithms and its characteristics which can further enhance signal processing and its efficiency respectively.

Luo C. et al in [11] determines the compression of sensing in multi-hop wireless sensor network for data collection in large-scale. The reduction of traffic in the network can be more effective and the load balancing can be maintained which can prolong the execution time of the network in this method. This author also addresses the random projection which is dense will not show execution enhancement of the networks that also furnishes the strategy for data collection of sensing in hybrid compression.

Vuran et al.[12] indicated in sensor networks, the addressed circumstances have high spatial and correlated temporally by sensors. The sparsity of sensor can results in these correlations with the sensor readings also with the wavelet transform with sparse requirements to satisfy the CS theory.

In [13] the authors address the framework of Compressive Data Gathering (CDG) to utilize the intersignals of sparsity in fashion of multi-hop in wide dense in the sensor networks. Matrix Completion (MC) is used to reconstruct full data matrix from part of its entries. In recent developments, E. J. candès et al. proves that this approach can recover missing entries from an incomplete set of entries, when the data matrix is at lower rank or at low rank matrix approximately.

Wang et al., in [14] indicates the scheme of energy efficiency: the random vector of sparse has been generated by each node. The adding up of vector from previous node(s) can result in next hop node for forwarding. The total number of communications by generating Sparse Random Projections (SRP) has been reduces by this scheme. Although, the frequent changes of routing paths leads to SRP, which are difficult to achieve in wireless sensor networks.

Zheng et al. [15] determines an approach on basis of CS correlated with the data gathering on random walk in WSNs, and the perspectives of graph theory and CS theory furnishes the mathematical based CS to utilize random walks. This work has been reconstructed for the sink node by original measurements for collecting all the data in the visited nodes in the last step by the random walk, and the number of independent random walks are the major focus with the length of each random walk, for collecting the collect random measurements this has been being required a sensor data k-sparse that can be reconstructed. The measure matrix will be modeled from the perspectives of bipartite graph theory, and ksparse signal gives the degree of reconstruction condition for a measure matrix.

Talari and Rahnavard [16] describes data persistence based C-storage for the techniques of CS and the nature of broadcast in wireless channel. However, this method of the proposed system usually minimizes the cost of transmission; the performance for reconstruction of CS is not proved actually. Specifically, DCT is only considered on the basis of sparsifying.

In [17], the authors represents the scheme which is novel and known as minimum transmission data gathering Tree (MTT), is determined to building of a CS measurement matrix based on the spanning tree and each node contribute its own data on the number of times to the measurements of CS. The scheme which is optimized for the cost of transmission has been introduced in this approach for multihop sensor networks using CS. Although the application of CS in many studies for sensor network, the investigation of CS application for sensor networks has been clustered.

Goel and Imielinski [18] proposed the concepts to monitor the data in energy-efficient way using MPEG compression in sensor networks. This can represent the mechanism called

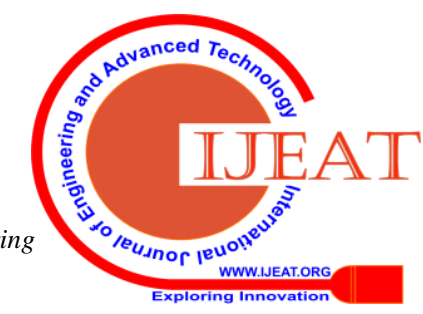


Prediction based Monitoring (PREMON) which is known to be best only for sending the difference between the present image and the prior image instead of the whole present image. The deviation due to the object motion in video between consecutive images has to deal by using MPEG.

In [19], the authors suggested an evolutionary algorithm of multi objective is to quantization process for generating combinations set for the corresponding parameters of various tradeoffs that are optimal among the performance of compression and loss of information. For the specific implementation, therefore, the combination with the user's most appropriate trade-off can be selected.

Datsika et al. [20] designed an enhancement of joint quality and on basis of Nash Bargaining solution for power control in Wireless Visual Sensor Networks(WVSNs). The proposed method for a cooperative multi hop for power control has been proceeds by Direct Sequence Code Division in Multiple Access for WVSN along with the resource allocation. The driven quality and resource allocation mechanism of power-aware is required for WVSN nodes by various resources necessitate. Both the function utilized for video quality and transmission power with the cost has been reflected on the basis of enhancement in joint quality and problem of power control. Increased fairness within the end to end quality between all the nodes has been attained by the function of Nash Bargaining.

Chen et al.[21] enhanced the compressed sensing by integrating the Spatio-temporal coding by clustered, by the network coding with the correlated data of sensor readings of spatiotemporal compression in the WSN. The recovery of original data with the increased probability has been enabled with the deployment of network coding and compressive sensing in real fields successfully. The lower reconstruction error has been provided in this scheme by the independent encoding for employing to all sensor nodes by containing the nodes in $\mathrm{CH}$ and can be decoded within sink nodes in order for the complexity of reduction. Furthermore, for determining a distributed algorithm to the iterative solution of optimizing the error of reconstruction in the compression scheme of clustered spatiotemporal can be optimized in this model.

The integration of the CS has been investigated in this work is the firstly and by the mechanism of diagonal matrix based block wise manner for clustering in WSNs. On the basis of CS clustering algorithm, we the sensors has been sampled and belongs to only once for each cluster and certain number of measurements has been generated by each cluster. The transmission of Compressive sensing measurements to the BS. The problem has been formulated and determined for minimum total energy consumption by the optimization by number of clusters.

\section{REASERCH METHODOLOGY}

The proposed methodology has data collection for energy efficient in wireless data sensing, without compromising the accuracy in sensing. The improvement of hierarchical compressive sensing (IHCS) with clustering approach using Block Tridiagonal Matrix (BTM) has been applied for the improvement of energy efficiency of the sensor nodes in the WSN without compromising the accuracy of data transmission. The formulated proposed work is as follows:
The formation algorithm of cluster has been initially designed to assign every sensor to elect them with a certain probability as gateway. On basis of probability of selection between the non-gateways nodes the $\mathrm{CH}$ nodes are selected in specific. The non- $\mathrm{CH}$ nodes are remains and are selected on the basis of their strength of signal. At the end, an approach of block tridiagonal matrix has been utilized on the basis of signal strength for selecting the selector nodes.

\section{A. Cluster Formation And Data Prediction}

The design of formation algorithm of cluster will elect every sensor themselves with a certain probability for assigning them as a gateway. Each $\mathrm{CH}$ have separate node to gateway from sink node. The $\mathrm{CH}$ of each cluster will select their own gateway node between the nodes by single hop that are nearer to sink node. Then the $\mathrm{CH}$ node can be a member of the cluster. A gateway selection (GWS) message has been sent by the $\mathrm{CH}$ to a gateway node, and hence the nodes that are selected should know whose gateway it is. The CHs and gateway nodes can perform the dissemination of query from a sink node and data gathering to sink node.

i). Energy considerations

Energy of the nodes initially in the range [Emin, Emax]

Each node's initial energy in the network,

$\mathrm{E}_{\mathrm{i}}=\mu_{\mathrm{i}} \mathrm{E}_{\min }(\mu \mathrm{i} \geq 1)$

So, total energy, $\mathrm{E}_{\text {total }}=\sum_{i=1}^{N} \mu_{i} E_{\text {min }}$

Energy dissipation in the $\mathrm{CH}$ node,

(2) $\mathrm{E}_{\mathrm{CH}}=(\mathrm{n} / \mathrm{k}) \mathrm{L} *\left[\mathrm{E}_{\mathrm{elec}}+\mathrm{E}_{\mathrm{da}}\right]+\mathrm{L}^{*} €_{\mathrm{fs}} * \mathrm{~d}_{\mathrm{BS}}^{2}$

Where, $\mathrm{k}=$ Cluster number, Eda= energy data aggregation, $\mathrm{d}_{\mathrm{BS}}=$ mean distance between $\mathrm{BS}$ and $\mathrm{CH}$

Energy dissipation in non- $\mathrm{CH}$ node,

$\mathrm{E}_{\mathrm{NCH}}=\mathrm{L}^{*}\left(\mathrm{E}_{\text {elec }}+\epsilon_{\mathrm{mp}} * \mathrm{~d}_{\mathrm{CH}}^{2}\right)$

(3) $\mathrm{E}_{\mathrm{T}}=\mathrm{E}_{\mathrm{CH}}+\mathrm{E}_{\mathrm{NCH}}$

Optimal number of clusters

$K_{o p t}=\sqrt{\frac{n}{2 \Pi}} * \sqrt{\frac{\epsilon_{f s}}{\epsilon_{m p}}} \frac{M}{d^{2}{ }_{B S}}$

(5)

Calculation of residual energy

$\mathrm{C}_{\text {Hprob }}(\mathrm{t})=\min \left[\left(\mathrm{E}_{\mathrm{i}(\mathrm{t})}\right) / \mathrm{E}_{\text {total }} \times \mathrm{k}, \mathrm{l}\right]$

Transmitted energy for one bit message over the distance $\mathrm{r}$ is given by:

$\mathrm{E}_{\text {trans }}(1, \mathrm{r})=\mathrm{E}_{\text {trans }}(\mathrm{x}$-elec $)+\mathrm{E}_{\text {trans-amp }}(1, \mathrm{r})$

The received energy by the amplifier is given by:

$\mathrm{E}_{\text {receive }}(\mathrm{r})=\mathrm{E}_{\text {receive-elec }}(\mathrm{r})+\mathrm{E}_{\text {elec }} * \mathrm{r}$

ii) Steps in $\mathrm{CH}$ selection

- Distinctive identifier (ID) will be allotted for each sensor node.

- The BS initiates the new round for the increment of the round number systematically.

- The $\mathrm{CHs}$ are selected on the basis of 0.1 probabilities and for each round the $\mathrm{CH}$ should not exceed 10 in number. For comparing the threshold value, the $\mathrm{CH}$ has been selected in random number to elect the $\mathrm{CH}$ itself for each round. 
- The gateway node has been selected after $\mathrm{CH}$ is formed; this remains closer to it.

The $\mathrm{CH}$ will generate clusters based on the minimum node distance by assigning the $\mathrm{CH}$ to each network sensor node. The sensor nodes wake up, senses information and transmits information to the appropriate CHs.

- $\quad$ All the cluster members will be receiving the $\mathrm{CHs}$ where the data are aggregated and the data are sent through single hop communication to the gateway nodes.

- $\quad$ The final data from gateway nodes will be sent to the $\mathrm{BS}$ and the process continues until the last round.

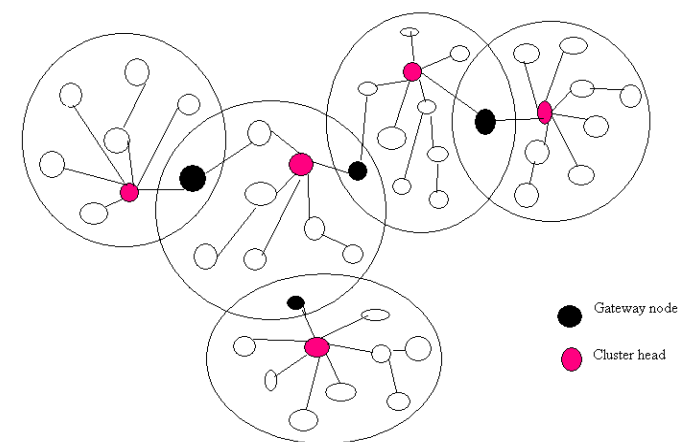

Fig. 2. Cluster formation and gate way node selection

\section{iii) Compressive sensing}

The proposed hierarchical compressive sensing uses cluster based compressive sensing leads to block diagonal matrices and the entries of the matrix are $\Phi 1$, Ф2, $\Phi 3, \ldots$ from each $\mathrm{CH}$. A tridiagonal matrix having the elements as block of square matrices, then The matrix is called a triangular block matrix ; the matrix is stacked if the upper or lower triangular blocks are off-diagonal. On basis of the iterative conjugation for block tridiagonal matrix with the sparse matrices are widely used as the direct approach until it is reduced to a tridiagonal form.

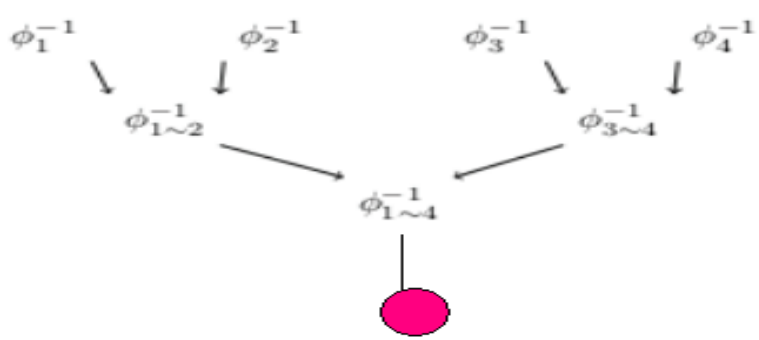

Fig. 3. Hierarchical compressive measurement

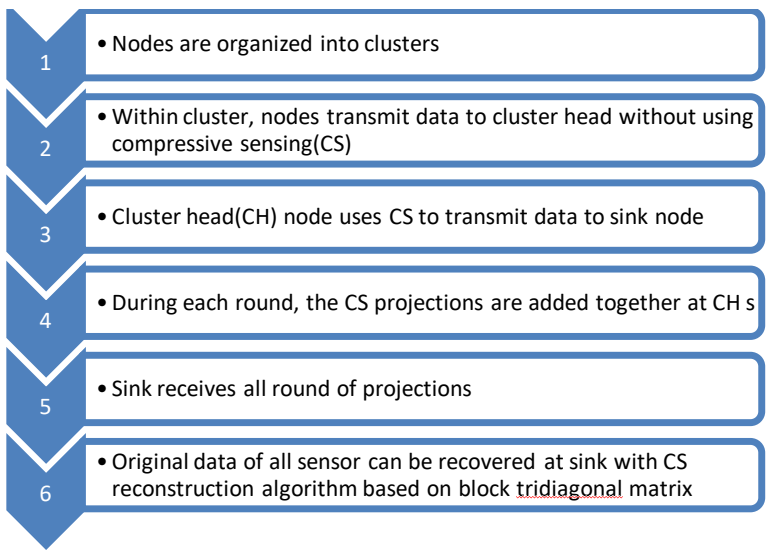

Fig. 4. Design flow diagram for proposed method
Hierarchical compressive sensing is performed at each $\mathrm{CH}$ and The block tridiagonal system of equation over the cluster $\mathrm{C} 1$ with the data $\mathrm{y} 1, \mathrm{y} 2, \mathrm{y} 3, \ldots . . \mathrm{yn}$ is given as,

$$
a_{v} y_{j}-1+b_{v} y_{j}+c_{v} y_{j}+1=d_{v}
$$

Where $\mathrm{a}, \mathrm{b}, \mathrm{c}$ are the compression factor, $\mathrm{v}$ is vertices from equation, $\mathrm{y}_{\mathrm{j}}$ is the data where $\mathrm{j}=\{1,2,3, \ldots \ldots \mathrm{n}\}$.

$$
\left[\begin{array}{c}
d 1 \\
d 2 \\
\cdot \\
\cdot \\
d N
\end{array}\right]=\left[\begin{array}{llll}
\phi 1 & & & \\
& \phi 2 & & \\
& & \cdots & \\
& & & \phi N
\end{array}\right]\left[\begin{array}{c}
y 1 \\
y 2 \\
\cdot \\
y N
\end{array}\right]
$$

The compression factor for each data can be implied by $\alpha=\left(a_{j}, b_{j}, c_{j}, d_{j}\right) \quad i \leq j \leq n$. Here we are considering the number of data as 6 . By using factorization, at zero condition upper and lower bound has been contained.

$$
\left\|\begin{array}{cccccc}
b 1 & c 1 & 0 & 0 & 0 & 1 \\
a 2 & b 2 & c 2 & 0 & 0 & 0 \\
0 & a 3 & b 3 & c 3 & 0 & 0 \\
0 & 0 & a 4 & b 4 & c 4 & 0 \\
0 & 0 & 0 & a 5 & b 5 & c 5 \\
1 & 0 & 0 & 0 & a 6 & b 6
\end{array}\right\|\left[\begin{array}{l}
y^{1} \\
y^{2} \\
y^{3} \\
y^{4} \\
y^{5} \\
y^{6}
\end{array}\right]=\left[\begin{array}{l}
d 1 \\
d 2 \\
d 3 \\
d 4 \\
d 5 \\
d 6
\end{array}\right]
$$

Where, ${ }_{1} \mathrm{~d}_{\mathrm{j}}$

$$
b_{j-1}^{*}=b_{j-1}-c_{j-1} b_{j-1} a_{j}, c_{j-1}^{*}=-c_{j-1} b_{j-1} c_{j}, d_{j-1}^{*}=d_{j-1}-c_{j-1} b_{j-}
$$

By Gaussian elimination, finally the compressed data is denoted as,

$$
\mathrm{Y}_{\mathrm{j}}=\alpha_{\mathrm{i}}+\left(\mathrm{y}_{\mathrm{j}-1} * \mathrm{y}_{\mathrm{j}}+1\right)
$$

\section{EXPERIMENTAL ANALYSIS}

The center of a distribution should be linked to some error measure with the context of any measure that has mean square error. The distance that has been taken for every data point Vertically from the point to the respective y value in the fit curve(error), and square the value. The compressive sensing implies the cellular data cost when using cellular networks. For emerging of crowd sensing and the application of compressive remains the major burden for network bandwidth. The following steps are applied to each node for data prediction strategy. Initially, the sensor node can be built on the basis of data from its model and measured values can be transmitted to the sink. By the model, the data predicted within the value tolerance of sensor nodes have been assumed by the operation of sink.

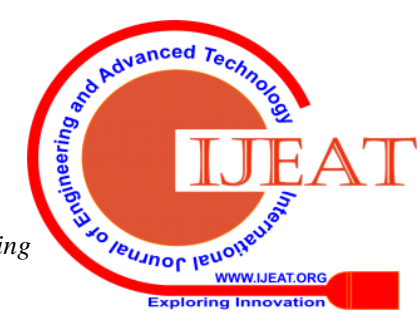




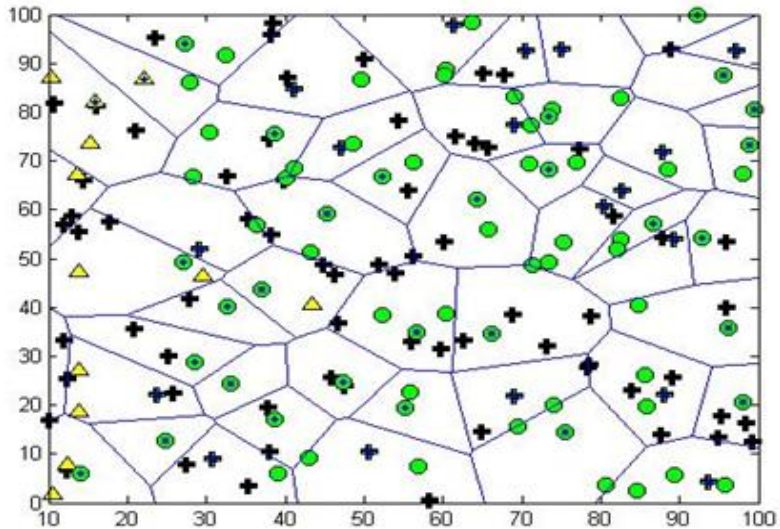

Fig. 5. Cluster formation in wireless sensor network

Fig.5. represents the formation of clustering in WSN. Here, we use MATLAB for simulating the network. We consider 100 nodes in $100 * 100$ square meter area with random locations of the nodes and the sink node is located at $(50,50)$ position in the middle of the region. After every iteration or round, the $\mathrm{CH}$ selection is changing dynamically based on the initial and residual energy of the nodes.

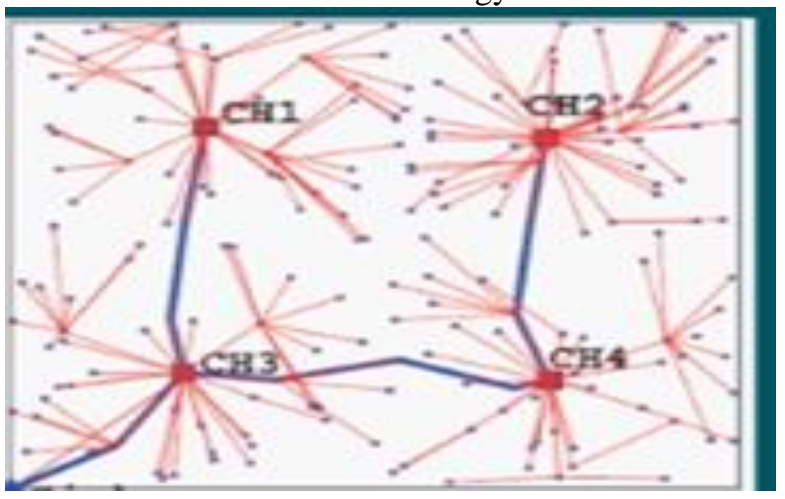

Fig. 6. Data transmision between clusters

Fig.6. represents the data transmission between among the nodes and also the data transmission between $\mathrm{CH}$ node and the member nodes. $\mathrm{CHs}$ will directly communicate with each other in order to aggregate the data to the final sink node or BS. The compressive sensing is applied at these $\mathrm{CH}$ nodes hierarchically and the orthogonal projections are added at each $\mathrm{CH}$. The compressed data with reduced data values at each $\mathrm{CH}$ is represented in a square matrix $\varnothing$. All these $\mathrm{CH}$ data are represented in the tridiagonal matrix as mentioned in section III(iii). Reconstruction of estimated data is obtained by applying the improved hierarchical compressive sensing algorithm at the sink node.

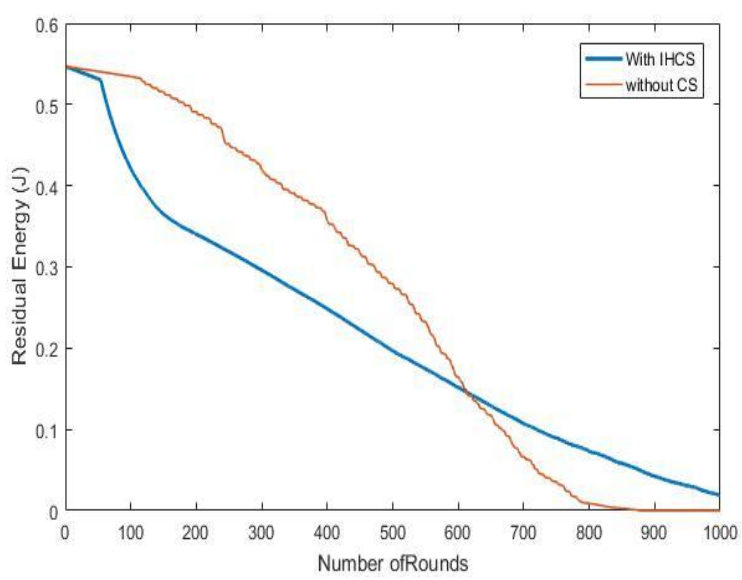

Fig. 7. Comparison with energy consumption
Fig.7. shows that at the end of 1000 rounds, the energy usage of the nodes is presented with proposed Cs and without CS in WSN with 100 nodes. From the above result, It shows that proposed method performs well with more residual energy.

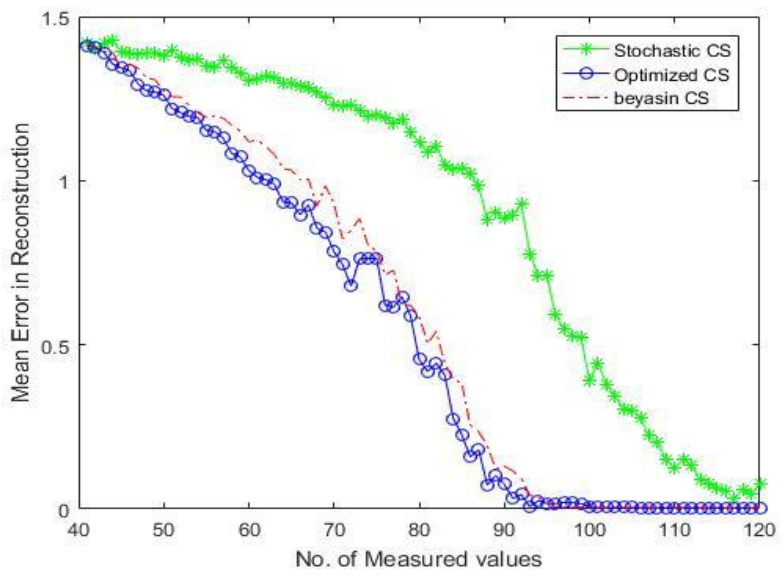

Fig.8. Comparison -proposed and other existing methods in terms of reconstruction error

Fig.8. demonstrates the comparison of the proposed technique with conventional compressive sensing techniques with regard to the amount of measurements in the reconstruction error. This shows that the proposed optimized CS method performs better in reduction of error and improving the accuracy in the sensing of the cluster based wireless sensor network.

\section{CONCLUSION}

Hence, this research paper determines the data collection method for energy efficient has been applied in the sensor network on the basis of integration for clustering and blockwise sensing improved hierarchical compressive sensing (IHCS) for enhancing the energy efficiency of the sensor nodes without reducing the accuracy of sensing with reduced reconstruction error. Based on the compressive sensing algorithm for the intermediate clustering produce the effective and efficient way to save energy. On basis of the minimizing communication requirement of energy of each sensor nodes have self-determination to which cluster they can be joined. The simulation will be carried out through MATLAB and the results were compared with the existing energy efficient algorithms. The novel approach of Integration of heterogeneous clustering in WSN with hierarchical compressive sensing with the proposed algorithm to provide the better result.

\section{REFERENCES}

1. K. Choi, J. Wang, L. Zhu, T. S. Sub, S. Boyd and L. Xing, "Compressive sensing based cone-beam computed tomography reconstruction with first order method," Medical Physics., vol. 37, pp.5113-5125, Sep, 2010.

2. Beutel, M. Dyer, R Lim, C. Plessl, M. Whorle, M. Yucel and L. Thiele, "Automated wireless sensor network testing," in Networked Sensing Systems, 2007. INNS ,07. Fourth International Conference on, 2007, pp.303-303

3. T. Ho, M. Medard, J. Shi, M. Effros, and D. R. Karge, "On randamized network coding," in Proc. 41 ${ }^{\text {st }}$ Annual Allerton Conference Communication, control and computing, 2003 
4. T. T. Do, G. Lu, N. H. Nguyen, and T. D. Tran, "Fast and efficient compressive sensing using structurally random matrices," IEEE Trans. Signal Process., vol. 60, no. 1, pp. 139-154, Jan. 2011.M. Young, The Technical Writer's Handbook. Mill Valley, CA University Science, 1989

5. M. F. Duarte and R. G. Baraniuk, "Kronecker compressive sensing," IEEE Trans. Image Process., vol. 21, no. 2, pp. 494-504, Feb. 2012.

6. B. Gong, P. Cheng, Z. Chen, N. Liu, L. Gui, and F. de Hoog, "Spatiotemporal compressive network coding for energy-efficient distributed data storage in wireless sensor networks," IEEE Commun. Lett., vol. 19, no. 5, pp. 1-1, May 2015.

7. J.Abouei,K.N.Plataniotis,S.Pasupathy,Green modulations in energy constrained wireless sensornetworks,IETCommun.5(2)(2011c)240251.

8. D.Takaishi,H.Nishiyama,N.Kato,R.Miura,Towardenergyefficientbigd atagatheringindenselydistributedsensornetworks,IEEETrans.Emerg.T op.Comput.2(3)(2014)388-397.

9. A.Ciancio,S.Pattem,A.Ortega,B.Krishnamachari,Energy efficient data representation and routing for wireless sensor networks based on a distributed wavelet compression algorithm ,in: Proceedings of IEEE International Conference on Information Processing in Sensor Networks, 2006, pp.309-316.

10. S. Feizi and M. Médard, "A power efficient sensing/communication scheme: Joint source-channelnetwork coding by using compressive sensing", CoRR, (2011), Monticello Israel.

11. C. Luo, F. Wu, J. Sun and C. Chen, "Efficient measurement generation and pervasive sparsity for compressive data gathering", Wireless Communications, IEEE Transactions, vol. 9, no. 12, (2010), pp. 3728-38.

12. MC Vuran, ÖB Akan, IF Akyildiz, "Spatio-temporal correlation: theory and applications for wireless sensor networks," Computer Networks, Vol.45, Issue.3, 2004, pp.245-259.

13. C. Luo, F. Wu, J. Sun, and C. W. Chen, "Compressive data gathering for large-scale wireless sensor networks," In Proceedings of MobiCom, 2009.

14. W. Wang, M. Garofalakis, and K. Ramchandran, "Distributed sparse random projections for refinable approximation," in Proceeding of 2007 International Symposium on Information Processing in Sensor Networks, IPSN, pp. 331-339.

15. H.Zheng, F. Yang, X. Tian, X. Gan, X. Wang, and S. Xiao, "Data gathering with compressive sensing in wireless sensor networks: A random walk based approach," IEEE Trans.Parallel Distrib. Syste., no.99,p.1, Feb.2014.

16. A. Talari, and N. Rahnavard, "Cstorage: Distributed data storage in wireless sensor networks emplying compressive sensing," in Proc. IEEE Global Telecommun. Conf., Dec. 2011, pp.1-5.

17. R. Xie and X. Jia, "Minimum transmission data gathering trees for compressive sensing in wireless sensor networks," in Global Telecommunications Conference (GLOBECOM 2011), 2011 IEEE, pp. $1-5$, Dec.

18. S.Goel, T.Imielinski, Prediction-based monitoring in sensor networks:t aking lessons from mpeg,ACMSIG COMMComput.Commun.Rev.31(5)(2001)82-98.

19. F.Marcelloni, M.Vecchio ,Enabling energy -efficient and lossy-aware data compression in wireless sensor networks by multi-objective evolutionary opti-mization, Inf.Sci.180(10)(2010)1924-1941.

20. Datsika, E.G., Katsenou, A. V., Kondi, L.P., Papapetrou, E. \& Parsopoulos, K.E. (2016). Joint Quality Enhancement and Power Control for Wireless Visual Sensor Networks based on the Nash Bargaining Solution. Digital Signal Processing. [Online]. 50. pp. 203-217. Available from: http://linkinghub.elsevier.com/retrieve/pii/S1051200416000038.

21. Chen, S., Zhao, C., Wu, M., Sun, Z., Zhang, H. \& Leung, V.C.M. (2016). Compressive network coding for wireless sensor networks: Spatio-temporal coding and optimization design. Computer Networks. [Online]. 108. pp. 345-356. Available from: http://linkinghub.elsevier.com/retrieve/pii/S138912861630.

\section{Authors Profile}

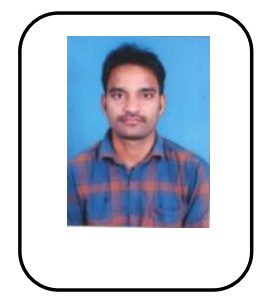

Venkat Rao Pasupuleti is currently an Assistant Professor in Lakireddy Balireddy College of Engineering. He received his B.Tech and M.Tech form JNTUK Kakinada in 2009 and 2012. He is an associate member of IETE. His research interests include Wireless Sensor Networks, Clustering, Compressive sensing, and optimization techniques.

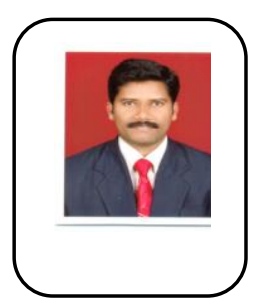

Ch. Balswamy is currently a Professor in Gudlavalleru Engineering College. He obtained his B.E from Andhra University and M.Tech from VTU, Belgaum. He received his PhD from J.N.T.U. College of Engineering, Anantpur in 2010. He has published more than 30 papers in international journals. His research interests include computer networks, mobile ad hoc networks. 